\title{
Hidden opportunities to reduce order fulfilment costs
}

\author{
Victoria Gerami ${ }^{1}$, and Ivan Shidlovskii ${ }^{2 *}$ \\ ${ }^{1}$ HSE University, Department of Logistics Systems and Management, 119049 Shabolovka st. 26, \\ Moscow, Russia \\ ${ }^{2}$ HSE University, Department of Logistics Systems and Management, 119049 Shabolovka st. 28/11, \\ Moscow, Russia
}

\begin{abstract}
The purpose of the work is to draw attention to the existing hidden reserves of increasing the efficiency of systems, in which solutions for their optimisation are associated with the analysis of models of problems of the theory of queueing networks (for example, problems of transport support for the supply of multiple orders). We are talking about such models that correlate precisely with the choice of queueing the existing portfolio of orders. Approaches to the optimisation of such systems are considered, allowing to consider the random nature of the change in the rates of fines over time and special additional costs related to the moment of order set formation.
\end{abstract}

\section{Introduction}

In recent decades, the development of logistics and operational management clearly shows that increasing efficiency for many companies is directly related to minimising associated costs. Moreover, the related problems can and conveniently be represented by models of the theory of queueing networks. Such models and their special modifications are often associated with optimising the order of execution of a given set of works (called in theory a portfolio of orders), for example, correlated with the transport provision of supplies of various goods. Some authors provide special models for evaluating the average lead times of make-to-order supply chains [7]. For the supply chain with the form postponement strategy is developed a two-stage tandem queueing network to characterise the correlation of the demand [10]. The problem of managing a homogeneous fleet of vehicles over time to serve a set of loads, each with a known origin and destination and a specified time window in which they must be served, is solved at [9]. Other approaches to modelling cash flows in the format of problems of a similar type can be found, in particular, in [3, 4]. At the same time, for particular tasks of choosing the order of performing the required operations, which correlate with the particular topic of the problem of "multi-armed bandits", the corresponding methods are presented, for example, in [5, 6].

At the same time, the possibility of taking into account the specifics of the corresponding cash flows for such tasks, in particular, and possible costs, in turn, undoubtedly, is a

* Corresponding author: shdlvsk-ivan@yandex.ru 
significant problem. Of course, this is because the optimisation of such cash flows can significantly affect the efficiency of the modelled supply chains. These models can be used as a still hidden and poorly studied reserve for increasing the efficiency of processes of this type.

Consider a special generalisation of the traditional model of the optimisation problem (see, for example, $[1,2]$ ) related to choosing the best order/priority of queueing portfolio orders when the financial result is specified based on the corresponding penalty functions. This generalisation will allow managers to consider both the random nature of the penalty rates and special costs that were not reflected in the original base model.

These are costs that can be attributed to each order. Indeed, in the general case, the orders of the formed portfolio could also differ in the already "running over" a priori financial costs or losses. For example, these costs could occur due to possible delays or disruptions concerning the required deadline for the delivery of such works if such deadlines have already been violated. In addition, the existing portfolio orders in the general case could also differ in the value of other additional costs, for example, correlated with the procedures for transferring these orders from one performer to another, in particular, due to the need for certain costs for the supply/transportation of specific materials or workpieces according to the specified orders. In this case, the model will need to take into account that such costs, in particular, will already take place immediately at the time of the portfolio formation.

\section{Results and discussion}

For an $i$-order (order number $i$ ) in the format of a more general model, in practice, it is also necessary to take into account those already correlated with such an order at the moment of portfolio formation (i.e., the moment of deciding on ordering orders in queues) specific additional costs. Let us denote such additionally taken into account losses for the $i$-order further by $\widetilde{\alpha}_{i}$. In this case, we will further consider the following generalised model for the traditional problem, which is initially associated with choosing the optimal order of queueing orders in a given portfolio. Suppose we are talking about an already formed portfolio of $\mathrm{N}$ available orders. In the format of the corresponding model, it is further assumed:

- $S_{i}$ - is the execution time of the $i$-order (these are independent random variables and with arbitrary laws of the probability distribution);

- $M\left(S_{i}\right)$ - known average expected values for $S_{i}(i=\overline{1, \mathrm{~N}})$;

- $\mu_{i}-$ is an indicator of the intensity of service of the $i$-order (determined by the equality $\left.\mu_{i}=1 / M\left(S_{i}\right)\right)$

- orders of the existing portfolio are served by one device;

- if queue of any order is started, then it is further implemented without interruption of the corresponding technological process;

- $c_{i}$ - rates of penalties that are charged for each unit of time spent in the i-order system for the traditional model $(i=\overline{1, \mathrm{~N}})$;

- $\tilde{c}_{i}$ - tariffs of fines, considered as random variables, just in the format of a modified model $(i=\overline{1, \mathrm{~N}})$;

- $\tilde{T}_{i}$ - moments of queue completion time for $i$-orders of the initially specified portfolio of orders (which, of course, in the general case, should be considered precisely as random variables); they are figuratively called the moments of "exit" of the $i$-order from the system $(i=\overline{1, \mathrm{~N}})$; 
- $\tilde{\alpha}_{i}$ - unique indicators of the expected additional total costs or losses for $i$-orders $(i=$ $\overline{1, N}$ ), depending only on the type of order, and, of course, in the general case, they can also be random variables; such costs are correlated precisely with the moment of formation of the portfolio.

We assume that the random variables $\tilde{c}_{i}$ for portfolio orders are pairwise independent. We also assume that the following specification of the simulated processes takes place. Random variables $\widetilde{c}_{i}$ do not depend on the duration $S_{j}(j \neq i)$ of the execution of other orders in the portfolio. In addition, they have arbitrary probability distribution laws with finite means, which we denote by $c_{i}$, i.e. $c_{i}=\mathrm{M}\left(\widetilde{c}_{i}\right)$. In other words, random rates of penalties $\widetilde{c}_{i}$, calculated for the $i$-order for each unit of time that the order is in the system (up to the moment of "exit" from the system after queue), are attributes of the corresponding order or a specific technology of processes of working with it.

The amount of total penalties associated with using a specific strategy, which will determine the order of selecting portfolio orders for queueing, is considered. Let us denote $\widetilde{C}$ the total value of such accumulated penalties on the time interval to execute all orders in the portfolio. The indicator $\tilde{C}$, of course, will be a random value. Accordingly, the total average expected losses for the entire portfolio of orders are analysed, denoted by $C$, where $C=M[\tilde{C}]$ is the mathematical expectation of the random variable $\tilde{C}$ we are interested in. Such a value (or, figuratively speaking, the "cost of stay" of orders in the queue) for the order portfolio is represented by the equality $C=M\left[\sum_{i=1}^{N} \tilde{c}_{i} \tilde{T}_{i}\right]$. Moreover, this indicator is minimised by choosing the order of queueing portfolio orders (see, for example, [2]).

With the considered modification of the model (taking into account additional losses $\widetilde{\alpha}_{i}$ for $i$-orders) for the average expected total losses, of course, during optimisation, one must also consider the expression $M\left[\sum_{i=1}^{N} \widetilde{\alpha}_{i}\right]$. The problem of minimising the costs of queueing a portfolio of orders will be presented in the form: $C(\bmod )=M\left[\sum_{i=1}^{N} \tilde{c}_{i} \tilde{T}_{i}\right]+M\left[\sum_{i=1}^{N} \tilde{\alpha}_{i}\right] \rightarrow \min$, moreover, minimisation should be achieved precisely and only by choosing the appropriate order of queueing portfolio orders.

To determine the optimal strategy, let us pay attention to the following feature of the above objective function. It is easy to see that the second term in the above expression for the modified indicator $C$ (mod) does not depend on the order in which the portfolio orders are selected for queue. Accordingly, during optimisation, it is necessary to consider the problem $C(\bmod )=M\left[\sum_{i=1}^{N} \tilde{c}_{i} \tilde{T}_{i}\right] \rightarrow$ min. Such a generalisation of the traditional problem of the theory of queueing networks was just considered in [2]. In particular, it was shown that the indicated modification of the optimisation problem is equivalent to a problem of a more straightforward form: $M\left[\sum_{i=1}^{N} c_{i} \tilde{T}_{i}\right] \rightarrow$ min. The specified feature of the objective function allows us to note the following accordingly.

The optimisation problem is reduced to the equivalent traditional formulation of the problem, which is considered in the theory of queuing networks $[2,8]$. Within the framework 
of the considered modification of the basic model of minimising the costs of queueing the portfolio of orders (moreover, taking into account the random tariffs of penalties, as well as special additional losses of the type indicated above), it will always turn out the following: for the optimal strategy of queueing portfolio orders must be performed in descending order (more precisely, not increasing) values of numeric indices of the $I_{i}=c_{i} \mu_{i}$. In other words, it is the strategy that is optimal, which in theory is called the optimal cu-rule without interruption.

\section{Conclusions}

Special modifications of the procedures for optimising the order of execution of portfolio orders, which were presented in this work, will optimise such solutions to ensure the following.

1. The possibility of considering the random nature of the penalties rates when modelling the total costs of fulfilling portfolio orders, as has been shown, is realised precisely by using the average values of $c_{i}$ for such penalties, where $c_{i}=\mathrm{M}\left(\tilde{c}_{i}\right)$.

2. The requirement to take into account special additional losses/costs, which must be correlated with each order of the portfolio, and, precisely at the time of the portfolio formation, does not affect the optimal strategy for selecting queueing orders.

3. The optimal order queueing strategy can be determined based on the traditional optimal $c \mu$-rules where the values of $c_{i}$ should be defined as the above indicators $c_{i}=\mathrm{M}\left(\tilde{c}_{i}\right)$.

4. The proposed approach to modifying the traditional optimal $c \mu$-rule (in the theory of queueing networks) will make it possible to expand the range of problems solved in modelling and optimising cash flows, correlated with the implementation of order portfolios.

5 . These models can be used as a still hidden and poorly studied reserve for increasing the efficiency of processes of this type

\section{References}

1. R.J. Boucherie and N.M. van Dijk, Queueing Networks: A Fundamental Approach, International Series in Operations Research \& Management Science, Springer, New York (2011)

2. G.L. Brodetskiy, Minimisation of Costs of Queueing of a Portfolio of Orders at Casual "Tariffs" of Penal Functions, RISK: Resources, Information, Supply, Competition, v.

3, pp. $96-102$ (2009)

3. F. Caro, J. Gallen, Dynamic Assortment with Demand Learning for Seasonal Consumer Goods. UCLA Anderson School of Management, Los Angeles, CA (2006)

4. S. Chick, N. Gans, An Economic Analysis of Simulation Selection Problems. OPIM Department Wharton University of Pennsylvania (2006)

5. N. Gans, G. Knox and R. Groson, Simple Models of Discrete Choice and Their Performance in Bandit Experiments. The Wharton School, University of Pennsylvania (2004)

6. J.C. Gittins, Bandit Processes and Dynamic Allocation Indices, J. R. Statist. Sos. v. 41(2) (1979)

7. N.R.S. Raghavan and N. Viswanadham, Generalized queueing network analysis of integrated supply chains, International Journal of Production Research, v. 39(2), pp. 205-224 (2001) 
8. J. Walrand, An introduction to queueing networks. Englewood Cliffs, N.J., Prentice Hall (1988)

9. P.B. Warren and C.A. Tassio, Dynamic Control of Logistics Queueing Networks for Large-Scale Fleet Management, Transportation Science, v. 32(2), pp. 90-109 (1998)

10. W. Zhou, W. Huang and R. Zhang, A two-stage queueing network on form postponement supply chain with correlated demands, Applied Mathematical Modelling, v. 38, p. 2734 (2014) 